\title{
Electro-orientation and electrorotation of metal nanowires
}

\author{
Juan J. Arcenegui, ${ }^{1}$ Pablo García-Sánchez, ${ }^{1}$ Hywel Morgan, ${ }^{2}$ and Antonio Ramos ${ }^{1, *}$ \\ ${ }^{1}$ Departamento Electrónica y Electromagnetismo, Facultad de Física, Universidad de Sevilla, Sevilla, Spain \\ ${ }^{2}$ School of Electronics and Computer Science, University of Southampton, Southampton, UK
}

(Received 27 September 2013; published 27 December 2013)

\begin{abstract}
The physical mechanisms responsible for the electrical orientation and electrical rotation of metal nanowires suspended in an electrolyte as a function of frequency of the applied ac electric field are examined theoretically and experimentally. The alignment of a nanowire in an ac field with a fixed direction is called electro-orientation. The induced constant rotation of a nanowire in a rotating electric field is called electrorotation. In both situations, the applied electric field interacts with the induced charge in the electrical double layer at the metal-electrolyte interface, causing rotation due to the torque on the induced dipole, and also from induced-charge electro-osmotic flow around the particle. First, we describe the dipole theory that describes electro-orientation and electrorotation of perfectly polarizable metal rods. Second, based on a slender approximation, an analytical theory that describes induced-charge electro-orientation and electrorotation of metal nanowires is provided. Finally, experimental measurements of the electro-orientation and electrorotation of metal nanowires are presented and compared with theory, providing a comprehensive study of the relative importance between induced-dipole rotation and induced-charge electro-osmotic rotation.
\end{abstract}

DOI: 10.1103/PhysRevE.88.063018

PACS number(s): 47.57.jd, 81.07.Gf, 87.15.Tt, 82.70.Dd

\section{INTRODUCTION}

Nanowires and nanotubes are extensively used in many areas of research and have attracted considerable attention for their potential used as sensors, building blocks in novel nanocircuits, or for the absorption of light in solar cells by means of a dense array of nanowires [1-3].

Electric fields are an appealing way of manipulating nanowires in solution because they are easily integrated into microdevices. However, despite the growing interest in the use of metal nanowires in nanotechnology the fundamental electrokinetic properties of metallic particles are poorly understood. The ac electrokinetic behavior of metal particles in electrolytes is commonly mistakenly modeled by considering the metal particle to be an isotropic lossy dielectric characterized by a finite conductivity and permittivity suspended in an electrolyte [4-7]. When a metal particle suspended in an electrolyte is subjected to an electric field, an electrical double layer is induced around the particle and this dominates both the dielectric properties and the electrokinetic behavior of the particle at low frequencies. Generally in the literature, the double-layer polarization of the metal-electrolyte interface is ignored, which leads to erroneous predictions of particle behavior. Ignoring the double layer predicts positive polarizabilities for all frequencies in the quasielectrostatic regime. This implies that there should not be any dipole relaxation for a metal particle and therefore fails to predict electrorotation of metallic particles as observed experimentally [8-11] or the frequency dependence of electro-orientation of metal rods as shown in this paper.

Here ac electrokinetics refers to the interaction of an ac electric field with a polarizable particle. Electrorotation (ROT) refers to the asynchronous rotation induced on a particle by a rotating electric field, while electro-orientation

\footnotetext{
*Corresponding author: ramos@us.es
}

$\left(\mathrm{EOr}^{1}\right)$ refers to the alignment of a nonspherical particle in an ac field with a fixed direction [12,13]. This paper describes experimental data and a full theoretical analysis of the electro-orientation and electrorotation of metal nanowires suspended in an electrolyte.

The metal nanowires in our experiments have typical lengths of several microns and diameters of hundreds of nanometers. These nanowires are particularly interesting for several reasons. First, the particle dimensions are larger that the thickness of the electrical double layer (DL) induced at the metal-electrolyte interface. For the electrolytes used in this work the DL thickness is in the range of 10 to $30 \mathrm{~nm}$. This condition allows us to use the thin double layer approximation. Second, the nanowires show noticeable Brownian motion. Although they are much denser than water and quickly sediment, the nanowires do not rest on the bottom of the device, but exhibit two-dimensional Brownian motion near the bottom and orientate freely when subjected to an electrical field. This occurs in low-conductivity electrolytes as used in this work where double-layer repulsion prevents adhesion to the bottom wall.

ROT experiments of these nanowires [11] show that their behavior can be understood using dipole theory with the assumption that the particles are perfectly polarizable (i.e., there are no Faradaic currents at the electrolyte/particle interface). The applied electric field produces an induced dipole on the particle. If the field is uniform, as in EOr and ROT, an electrical torque acts on this induced dipole. If the field is nonuniform, as in dielectrophoresis (DEP), then the induced dipole experiences a force which moves the particle up or down field gradients. For perfectly polarizable particles there is a characteristic frequency $\omega_{\mathrm{RC}}$ which is the reciprocal of the resistor-capacitor (RC) characteristic time for charging the capacitance of the particle/electrolyte

\footnotetext{
${ }^{1}$ We use the abbreviation EOr to make a distinction with EO for electro-osmosis
} 

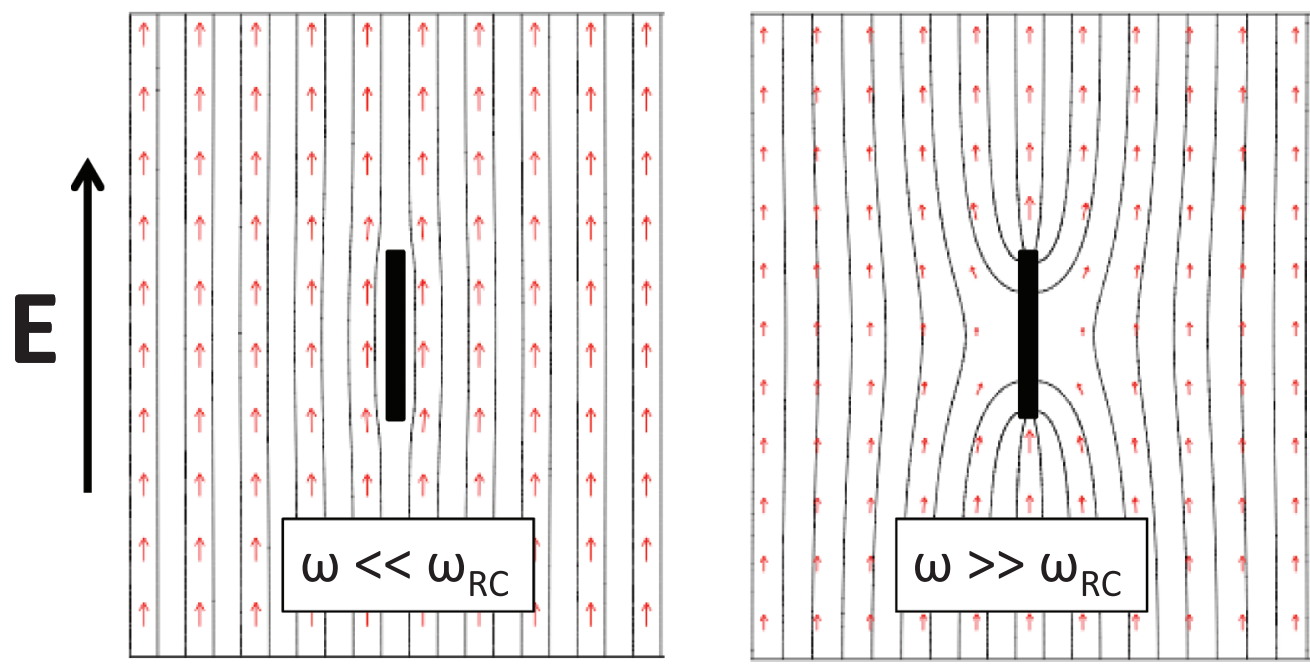

FIG. 1. (Color online) Illustration of the electric field lines for low and high frequencies of the applied ac electric field around a metal rod suspended in an electrolyte. The electric field is applied in the axial direction.

double layer through the resistance of the bulk electrolyte. For instance, the characteristic frequency for a sphere of radius $a$ is $\omega_{\mathrm{RC}}=\sigma /\left(C_{\mathrm{DL}} a\right)$ [9], where $\sigma$ is the conductivity of the electrolyte and $C_{\mathrm{DL}}$ is the double-layer capacitance per unit area. For rods this characteristic frequency depends on the aspect ratio. For angular frequencies $\omega$ of the applied electric field much smaller than $\omega_{\mathrm{RC}}$, ions driven by the electric field accumulate at the interface inducing a double layer. The electric field lines surround the particle as shown in Fig. 1. From the perspective of an observer the particle behaves as an insulator at these frequencies. For frequencies much greater than $\omega_{\mathrm{RC}}$, there is insufficient time to charge the double-layer capacitance, there is negligible induced charge in the Debye layer, and the particle behaves as a perfect conductor. In this case the electric field lines intersect perpendicularly to the surface of the particle as shown in the figure.

It is also known that at low frequencies the interaction of the applied electric field with the induced charges in the double layer can give rise to electro-osmotic fluid flow around the particle, so-called induced-charge electro-osmosis (ICEO), and this flow can lead to rotational motion of the particle [14-16]. In the ROT experiments presented in our previous paper [11], the amplitude of this effect on the behavior of the particles could not be quantified. However, it was observed that the effect of this ICEO flow was much smaller than the rotation due to the torque on the dipole (see also below). Importantly, the EOr experiments provide a means of separating the two mechanisms: at low frequencies ICEO flow around the nanowire will orientate it with the electric field, while at high frequencies the nanowire orientation is caused by the electrical torque on the induced dipole. This is because at high frequencies $\left(\omega \gg \omega_{\mathrm{RC}}\right)$ there is negligible charge in the double layer and therefore negligible ICEO flow. The particle behaves as a conductor and the dipole is maximum, so the particle is oriented only by the electrical torque. At low frequencies the particle behaves as an insulator. The induced dipole of a slender insulating rod is negligible compared to that of a conducting rod. At low frequencies the electric field lines deviate very little as shown in Fig. 1, which means that the induced dipole is very small. Since the double layer is fully charged, ICEO flow dominates [15,17].

This article is organized as follows. We begin by describing dipole theory that underpins EOr and ROT of perfectly polarizable metal rods. Section II B provides an analytical theory to describe induced-charge EOr and ROT as a function of frequency for slender cylindrical particles. This theory builds on the existing theory for low-frequency limits $[14,15,18]$. Experimental observations and measurements of the EOr and ROT of silver nanowires are described in Sec. III and the data compared with theory in Sec. IV, followed by conclusions.

\section{THEORETICAL ANALYSIS}

\section{A. Dipole theory}

Consider a perfectly polarizable metal cylinder suspended in an electrolyte and subjected to an ac electric field of angular frequency $\omega$ as shown in Fig. 2. The radius of the cylinder is $b$ and its length is $2 a$. The cylinder is polarized by the action of the applied electric field. Using phasors, the induced electrical dipole on the cylinder can be written as

$$
\mathbf{p}=\alpha_{z} \mathbf{E}_{z}+\alpha_{x} \mathbf{E}_{x}
$$

where $\mathbf{E}_{z}$ and $\mathbf{E}_{x}$ are the electric field components parallel and perpendicular to the cylinder axis, and $\alpha_{z}, \alpha_{x}$ are the corresponding complex polarizabilities. The time-averaged electrical torque on the induced dipole is

$$
\boldsymbol{\tau}_{e}=\frac{1}{2} \operatorname{Re}\left[\mathbf{p} \times \mathbf{E}^{*}\right]=\mathbf{u}_{y} \frac{1}{2} \operatorname{Re}\left[\alpha_{z} E_{z} E_{x}^{*}-\alpha_{x} E_{x} E_{z}^{*}\right],
$$

where * means complex conjugate. In EOr, the applied electric field is of the form

$$
\mathbf{E}(t)=E_{0} \operatorname{Re}\left[\left(\cos \theta \mathbf{u}_{z}+\sin \theta \mathbf{u}_{x}\right) e^{i \omega t}\right],
$$

which leads to a torque on the particle given by

$$
\tau_{e}=\frac{E_{0}^{2}}{2} \operatorname{Re}\left[\alpha_{z}-\alpha_{x}\right] \cos \theta \sin \theta .
$$




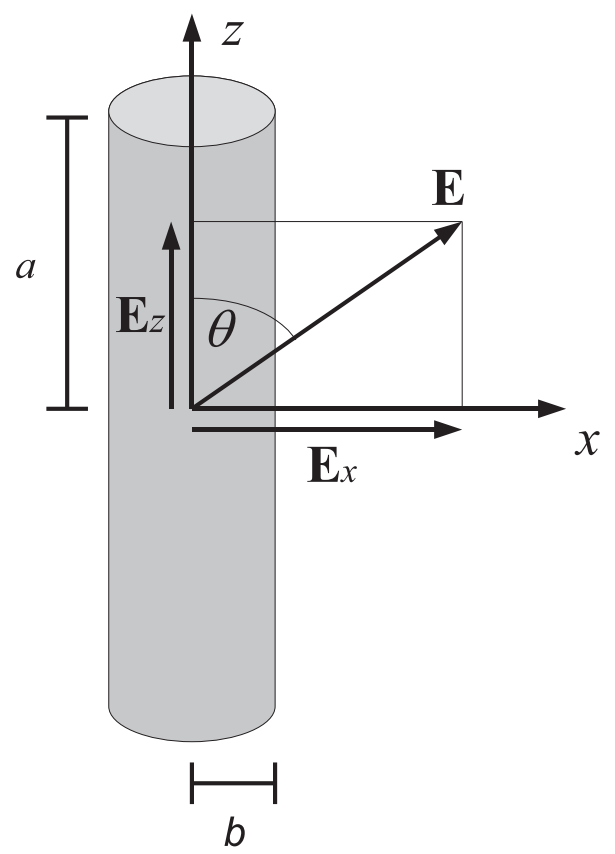

FIG. 2. Cylinder in an electric field.

In ROT, the applied electric field is of the form

$$
\mathbf{E}(t)=E_{0} \operatorname{Re}\left[\left(\mathbf{u}_{z}-i \mathbf{u}_{x}\right) e^{i \omega t}\right],
$$

which leads to a torque on the particle

$$
\tau_{e}=-\frac{E_{0}^{2}}{2} \operatorname{Im}\left[\alpha_{z}+\alpha_{x}\right] .
$$

These equations show that the EOr and ROT torques are proportional to the real and imaginary parts of the polarizabilities, respectively. For slender particles the absolute polarizability along the long axis is much greater than along the short axis $\left(\left|\alpha_{z}\right| \gg\left|\alpha_{x}\right|\right)$ and, therefore, the EOr and ROT torques are approximately proportional to $\operatorname{Re}\left[\alpha_{z}\right]$ and $\operatorname{Im}\left[\alpha_{z}\right]$, respectively [11].

To obtain the polarizabilty $\alpha_{z}$ we need to solve the potential of a perfectly polarizable cylinder suspended in an electrolyte subjected to an applied electric field. The thin double layer approximation allows us to describe the bulk electrolyte as an ohmic liquid where the charge conservation equation can be written as $\nabla \cdot \mathbf{j}=\nabla \cdot[(\sigma+i \omega \varepsilon) \mathbf{E}]=0$, where $\sigma$ and $\varepsilon$ are, respectively, the electrical conductivity and dielectric permittivity of the electrolyte. For a homogeneous liquid the potential phasor satisfies Laplace's equation $\nabla^{2} \Phi=0$. For a perfectly polarizable particle, i.e., there is no charge transfer across the interface, the electrical current from the bulk charges the electrical double layer. The latter is modeled as a capacitor with specific capacitance $C_{\mathrm{DL}}$. This results in the following boundary condition for $\Phi$ on the particle surface $S$ [19-21]:

$$
\left.\sigma(\mathbf{n} \cdot \nabla \Phi)\right|_{S}=\left.i \omega C_{\mathrm{DL}}(\Phi-V)\right|_{S}=\left.i \omega C_{\mathrm{DL}} \Phi\right|_{S},
$$

where $\sigma$ is the liquid conductivity, $\mathbf{n}$ is a vector normal to the particle surface, $\Phi$ is the potential just outside the double layer, and $V$ is the potential in the metal rod, which is taken as zero without loss of generality. The electric

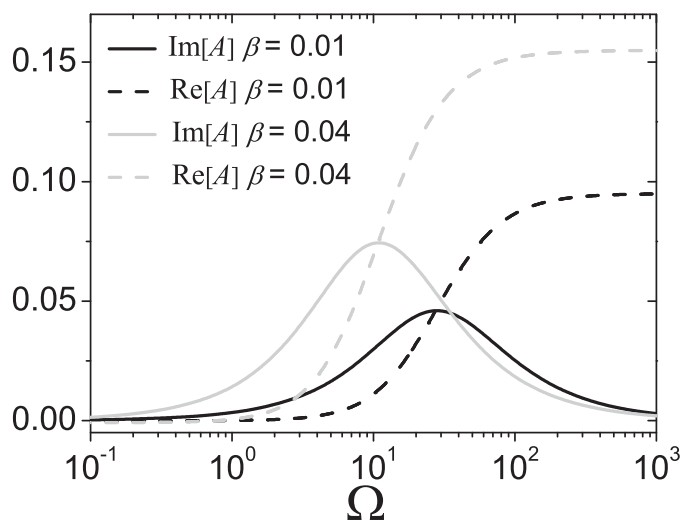

FIG. 3. Real and imaginary nondimensional polarizability $A$ as a function of nondimensional frequency $\Omega$ for particle aspect ratio $\beta=0.04$ and $\beta=0.01$.

potential was numerically calculated using the commercial finite element solver COMSOL. The polarizability as a function of applied signal frequency is obtained from the second term of the multipolar expansion of the electric potential [22]. Reference [11] provides a detailed description of how the polarizability for a cylinder with given aspect ratio $\beta$ is calculated.

The previous boundary condition allows us to define a characteristic frequency. Taking the rod semilength $a$ as a characteristic length scale, dimensional analysis leads to $\omega_{0}=\sigma /\left(a C_{\mathrm{DL}}\right)$. The typical frequency $\omega_{\mathrm{RC}}$, which is the reciprocal of the $\mathrm{RC}$ time for charging the double layer, should be $\omega_{\mathrm{RC}}=\omega_{0} g(\beta)$, where $g(\beta)$ is a nondimensional function of the aspect ratio $\beta=b / a$. As stated previously, for frequencies $\omega \ll \omega_{\mathrm{RC}}$, the double layer is fully charged and the particle behaves as an insulator. For frequencies $\omega \gg \omega_{\mathrm{RC}}$ the particle behaves as a conductor because there is negligible charge in the double layer. This implies that the real part of both polarizabilities $\alpha_{z}$ and $\alpha_{x}$ increases from negative to positive values as the frequency increases. In this situation, theory predicts that the imaginary part is zero at high and low frequencies with a positive single maximum at $\omega_{\mathrm{RC}}$. Given that the imaginary part is positive, Eq. (6) predicts that the particle rotates counterfield in a rotating field; i.e., particle rotation is in the opposite direction to the electric field. Since nanorods are slender, $\left|\alpha_{z}\right| \gg\left|\alpha_{x}\right|$, we focus on computing only $\alpha_{z}$.

Figure 3 shows the real and imaginary parts of nondimensional polarizability $A=\alpha_{z} /\left(4 \pi a^{3} \varepsilon\right)$ as a function of nondimensional frequency $\Omega=\omega / \omega_{0}$ for particles with aspect ratio $\beta=b / a=0.04$ (nanowires used in this work) and $\beta=0.01$. It can be seen that the nondimensional polarizability is a function of this aspect ratio $\beta$. We can also see that the real part of the polarizability at low frequencies is much smaller than the real part of the polarizability at high frequencies, meaning that when the particle behaves as an insulator (low $\omega)$ the magnitude of the induced dipole is much smaller than when the particle behaves as a conductor (high $\omega$ ).

From the time-average electrical torque, the rotation speed $\dot{\theta}$ of the nanowires can be determined, provided the rotational viscous friction coefficient $\gamma_{\infty}$ is known. Broersma [23,24] provides the following expression for the viscous coefficient 
of a cylinder suspended in a liquid of viscosity $\eta$ :

$$
\gamma_{\infty}=\frac{8 \pi a^{3} \eta}{3 f(\lambda)}
$$

where $\lambda=\ln (2 a / b)$ and

$$
f(\lambda)=\lambda-\left(1.14+0.2 / \lambda+16 / \lambda^{2}-63 / \lambda^{3}+62 / \lambda^{4}\right) .
$$

This equation holds for a cylinder that rotates around the axis passing through its center and perpendicular to the cylindrical axis. Balancing the electrical and viscous torques $\tau_{e}=\gamma_{\infty} \dot{\theta}$, the steady state rotation speed in ROT is

$$
\dot{\theta}=\frac{3 f(\lambda) E_{0}^{2} \varepsilon \operatorname{Im}[A(\omega)]}{4 \eta}
$$

and in EOr

$$
\dot{\theta}=\frac{3 f(\lambda) E_{0}^{2} \varepsilon \operatorname{Re}[A(\omega)] \sin \theta \cos \theta}{4 \eta} .
$$

The main predictions of dipole theory in terms of EOr and ROT of metal nanowires are (a) counterfield rotation with a single ROT peak, (b) strong orientation of the nanowires at high frequency and weak orientation at low frequency for EOr, and (c) both ROT and EOr show the same characteristic frequency $\omega_{\mathrm{RC}}=\sigma / a C_{\mathrm{DL}} g(\beta)$. This frequency is of the order of the reciprocal RC time for charging the double-layer capacitance of the particle/electrolyte surface through an electrolyte.

\section{B. Slender metal rods subjected to ac electric fields}

The main objective of this section is to analyze the rotational motion of micro- or nanowires due to induced-charge electroosmotic (ICEO) flow around the particle [14-16]. The analysis is based on the assumption of a slender body and thin double layer approximation. The analysis also provides the rotational motion due to the torque on the induced dipole. The complete rotation of the particle can be written as the rotation due to the ICEO flow around the particle plus the rotation due to the external torque (see, for instance, Ref. [15]).

Consider the same geometry as shown previously (Fig. 2). Using superposition, the total electrical potential outside the cylinder can be written as the sum of the potentials $\Phi_{a}$ and $\Phi_{b}$ corresponding, respectively, to the potentials generated by $\mathbf{E}_{a}=E_{0 a} \mathbf{u}_{z}$ and $\mathbf{E}_{b}=E_{0 b} \mathbf{u}_{x}$.

\section{Electric field along axial direction}

Using phasors, the applied electric field is $\mathbf{E}_{a}=E_{0 a} e^{i \omega t} \mathbf{u}_{z}$. This electric field induces a distribution of charges on the cylinder. The electric potential outside the cylinder can be written in cylindrical coordinates $(\rho, z)$ as if it were created by an effective linear charge density $\lambda(z)$ along the $z$ axis [25-27]:

$$
\Phi_{a}=-E_{0 a} z+\frac{1}{4 \pi \varepsilon} \int_{-a}^{a} \frac{\lambda\left(z^{\prime}\right)}{\sqrt{\left(z-z^{\prime}\right)^{2}+\rho^{2}}} d z^{\prime} .
$$

In order to apply this slender-body approximation, we imagine that the rods (nanowires) are cylinders with rounded ends. In our case the linear charge density $\lambda(z)$ is complex, creating in-phase and out-of-phase perturbed potentials. The boundary condition at the surface of the cylinder accounts for the conservation of charge: the bulk current arriving at the cylinder surface charges the double-layer capacitance. Outside the region near the ends $z= \pm a$, we have at $\rho=b$

$$
\left.i \omega C_{\mathrm{DL}} \Phi_{a}\right|_{\rho=b}=\left.\sigma \frac{\partial \Phi_{a}}{\partial \rho}\right|_{\rho=b} .
$$

For a slender cylinder where $\lambda(z)$ varies over a length $a$ much larger than the radius $b$, the potential at the surface is [27]

$$
\Phi_{a}(z, b) \sim-E_{0 a} z+\frac{\lambda(z)}{2 \pi \varepsilon} \ln (a / b) .
$$

Here the integral has been evaluated asymptotically for $b \ll$ $a$, which produces a logarithmically large contribution. The latter is recognized as the potential close to a line charge $\Phi \sim$ $-(\lambda / 2 \pi \varepsilon) \ln \rho$. We apply again the slender approximation to the radial derivative of potential at $\rho=b$

$$
\left.\frac{\partial \Phi_{a}}{\partial \rho}\right|_{b}=-\frac{1}{4 \pi \varepsilon} \int_{-a}^{a} \frac{\lambda\left(z^{\prime}\right) b}{\left(\left(z-z^{\prime}\right)^{2}+b^{2}\right)^{3 / 2}} d z^{\prime} \sim-\frac{\lambda(z)}{2 \pi \varepsilon b} .
$$

This equation is essentially the application of Gauss's law, where the radial component of the electric field is related to the linear charge density. Substituting (14) and (15) into the boundary condition (13) gives the linear charge density

$$
\lambda(z)=2 \pi \varepsilon E_{0 a} z \frac{i \Omega_{b}}{1+i \Omega_{b} \ln (1 / \beta)},
$$

where $\beta^{-1}=a / b$ and $\Omega_{b}=C_{\mathrm{DL}} \omega b / \sigma$ is a nondimensional frequency related to the previous nondimensional frequency by $\Omega_{b}=\Omega \beta$. The problem for $\lambda(z)$ near the ends $z= \pm a$ remains. A small correction is expected at the ends of order $b / a$, but this is negligible compared to $1 / \ln (1 / \beta)$ [27].

The induced dipole is

$$
p_{z}=\int_{-a}^{a} z \lambda(z) d z=\frac{4 \pi \varepsilon a^{3} E_{0 a}}{3} \frac{i \Omega_{b}}{1+i \Omega_{b} \ln (1 / \beta)} .
$$

The real and imaginary parts of nondimensional polarizability $A=\alpha_{z} /\left(4 \pi a^{3} \varepsilon\right)$ are

$$
\begin{aligned}
& \operatorname{Re}[A]=\frac{\Omega_{b}^{2} \ln (1 / \beta)}{3\left[1+\Omega_{b}^{2} \ln ^{2}(1 / \beta)\right]}, \\
& \operatorname{Im}[A]=\frac{\Omega_{b}}{3\left[1+\Omega_{b}^{2} \ln ^{2}(1 / \beta)\right]} .
\end{aligned}
$$

From this, the characteristic frequency (maximum of imaginary part) is $\Omega_{b}=1 / \ln (1 / \beta)$. For $\Omega_{b} \rightarrow \infty$, the cylinder behaves as a perfect conductor and the polarizability is in accordance to the known result for a slender conducting spheroid $\alpha=4 \pi \varepsilon a^{3} /[3 \ln (1 / \beta)]+O\left(1 / \ln ^{2}(1 / \beta)\right)$ [12]. For $\Omega_{b} \rightarrow 0$, the cylinder behaves as a perfect insulator and the polarizability goes to 0 in this approximation. In relation to this, the polarizability of a slender perfect insulating spheroid is of the order of $\beta^{2} \ln (1 / \beta)$ smaller than that of a conducting spheroid [12]. The Appendix compares these functions obtained using the slender-body approximation with polarizabilities computed numerically. Although the convergence is slow [it should go as $1 / \ln (1 / \beta)$ ], the slender approximation is providing the correct trends and proper order of magnitude for the polarizability. Therefore, the slender-approximation estimate for the characteristic frequency, $\Omega_{b}=1 / \ln (1 / \beta)$, is 
in dimensional form $\omega_{\mathrm{RC}}=\sigma / b C_{\mathrm{DL}} \ln (a / b)$, while the order of magnitude for the polarizability is $\left|\alpha_{z}\right| \sim 4 \pi \varepsilon a^{3} / 3 \ln (a / b)$.

\section{Electric field perpendicular to the axis}

Assume that the applied electric field is $\mathbf{E}_{b}=E_{0 b} \mathbf{u}_{x}$ Neglecting edge effects, the electric potential near the slender cylinder can be written in polar coordinates $(\rho, \phi)$ as

$$
\Phi_{b}=-E_{0 b} \rho \cos \phi+\left(\frac{i \Omega_{b}-1}{i \Omega_{b}+1}\right) \frac{E_{0 b} b^{2} \cos \phi}{\rho},
$$

where $i \omega C_{\mathrm{DL}} \Phi_{b}=\sigma\left(\partial \Phi_{b} / \partial \rho\right)$ at $\rho=b$ has been taken into account. The term proportional to $1 / \rho$ is the potential due to the $2 \mathrm{D}$ dipole

$$
p_{\ell}=2 \pi \varepsilon E_{0 b} b^{2} \frac{i \Omega_{b}-1}{i \Omega_{b}+1},
$$

which multiplied by the cylinder length $2 a$ gives the induced 3D dipole, neglecting edge effects,

$$
p_{x}=4 \pi \varepsilon E_{0 b} a b^{2} \frac{i \Omega_{b}-1}{i \Omega_{b}+1} .
$$

This dipole $p_{x}$ is of the order of $\beta^{2} \ln (1 / \beta)$ smaller than $p_{z}$.

\section{Rotation due to electrical torque}

The time-averaged torque on the induced dipole is [see Eq. (2)]

$$
\begin{aligned}
\boldsymbol{\tau}_{e} & =\frac{1}{2} \operatorname{Re}\left[\left(\mathbf{p}_{z}+\mathbf{p}_{x}\right) \times\left(\mathbf{E}_{0 a}+\mathbf{E}_{0 b}\right)^{*}\right] \\
& \approx \frac{2 \pi \varepsilon a^{3}}{3} \operatorname{Re}\left[\frac{i \Omega_{b}}{1+i \Omega_{b} \ln (1 / \beta)} E_{0 a} E_{0 b}^{*}\right] \mathbf{u}_{y},
\end{aligned}
$$

where we have neglected a contribution of the order of $(b / a)^{2}$. The EOr torque is [see Eq. (4)]

$$
\boldsymbol{\tau}_{e}=\frac{2 \pi \varepsilon a^{3}}{3} \frac{\Omega_{b}^{2} \ln (1 / \beta)}{1+\Omega_{b}^{2} \ln ^{2}(1 / \beta)} E_{0}^{2} \cos \theta \sin \theta \mathbf{u}_{y} .
$$

This torque tends to align the cylindrical particle with the applied field. The angular velocity, taking the viscous coefficient for slender body equal to $8 \pi \eta a^{3} / 3 \ln (1 / \beta)$, becomes

$$
\dot{\theta}=\frac{\varepsilon E_{0}^{2}}{4 \eta} \frac{\Omega_{b}^{2} \ln ^{2}(1 / \beta)}{1+\Omega_{b}^{2} \ln ^{2}(1 / \beta)} \cos \theta \sin \theta .
$$

For $\Omega_{b} \rightarrow 0$ the angular velocity is zero in this approximation [the actual value is of order $(b / a)^{2}$ from a contribution of the dipole along $x$ ]. For $\Omega_{b} \rightarrow \infty$, the angular velocity is $\dot{\theta}=$ $(\varepsilon / 4 \eta) E_{0}^{2} \cos \theta \sin \theta$, in accordance with the known solution for a slender conducting spheroid $\dot{\theta}=(\varepsilon / 4 \eta) E_{0}^{2} \cos \theta \sin \theta+$ $O(1 / \ln (1 / \beta))$ [12] [notice that there is a $1 / 2$ factor due to the time average of $\left.\cos ^{2}(\omega t)\right]$.

The ROT torque is [see Eq. (6)]

$$
\boldsymbol{\tau}_{e}=-\frac{2 \pi \varepsilon a^{3}}{3} \frac{\Omega_{b}}{1+\Omega_{b}^{2} \ln ^{2}(1 / \beta)} E_{0}^{2} \mathbf{u}_{y}
$$

and the angular velocity is

$$
\dot{\theta}=-\frac{\varepsilon E_{0}^{2}}{4 \eta} \frac{\Omega_{b} \ln (1 / \beta)}{1+\Omega_{b}^{2} \ln ^{2}(1 / \beta)} .
$$

The minus sign indicates that the particle rotates in the opposite direction to the rotating applied field (counterfield rotation), with a maximum rotation speed equal to $|\dot{\theta}|_{\max }=\varepsilon E_{0}^{2} / 8 \eta$ at $\Omega_{b}=1 / \ln (1 / \beta)$.

\section{Rotation due to induced-charge electro-osmotic flow}

Having determined the electrical potential, the timeaveraged slip velocity on the surface of the particle can be calculated, which is [28]

$$
\mathbf{v}_{s}=-(\varepsilon / 2 \eta) \operatorname{Re}\left[\Phi_{s} \nabla_{s} \Phi^{*}\right],
$$

where $\Phi_{s}$ is the potential on the surface of the particle and $\nabla_{s}$ stands for surface gradient. Taking into account that the total potential is $\Phi=\Phi_{a}+\Phi_{b}$ :

$$
\begin{gathered}
\Phi_{s a}=\frac{-E_{0 a} z}{1+i \Omega_{b} \ln (1 / \beta)}, \\
\nabla_{s} \Phi_{a}=\frac{-E_{0 a}}{1+i \Omega_{b} \ln (1 / \beta)} \mathbf{u}_{z}, \\
\Phi_{s b}=-\frac{2 E_{0 b} b \cos \phi}{1+i \Omega_{b}}, \\
\nabla_{s} \Phi_{b}=\frac{2 E_{0 b} \sin \phi}{1+i \Omega_{b}} \mathbf{u}_{\phi} .
\end{gathered}
$$

Because of symmetry, the terms $\Phi_{s a} \nabla_{s} \Phi_{a}^{*}$ and $\Phi_{s b} \nabla_{s} \Phi_{b}^{*}$ do not generate rotation and therefore we only consider the crossed terms $\Phi_{s a} \nabla_{s} \Phi_{b}^{*}$ and $\Phi_{s b} \nabla_{s} \Phi_{a}^{*}$. Following references $[15,29]$ the contribution to the angular velocity of a slender particle due to slip velocity on the surface is

$$
\dot{\theta}=-\frac{3}{2 a^{3}} \int_{-a}^{a} z \mathbf{u}_{z} \times \tilde{\mathbf{v}}_{s} d z,
$$

where

$$
\tilde{\mathbf{v}}_{s}=\frac{1}{2 \pi} \int_{0}^{2 \pi} \mathbf{v}_{s}(z, \phi) d \phi .
$$

In our case the term $\Phi_{s b} \nabla_{s} \Phi_{a}^{*}$ is parallel to $\mathbf{u}_{z}$ and does not produce rotation. The term of the slip velocity that does produce rotation is

$$
\begin{aligned}
\mathbf{v}_{s}^{\text {rot }} & =-\frac{\varepsilon}{2 \eta} \operatorname{Re}\left[\frac{-E_{0 a} z}{1+i \Omega_{b} \ln (1 / \beta)} \frac{2 E_{0 b}^{*} \sin \phi}{1-i \Omega_{b}}\right] \mathbf{u}_{\phi} \\
& \approx \frac{\varepsilon}{2 \eta} \operatorname{Re}\left[\frac{2 z E_{0 a} E_{0 b}^{*} \sin \phi}{1+i \Omega_{b} \ln (1 / \beta)}\right] \mathbf{u}_{\phi},
\end{aligned}
$$

where the last approximation comes from the fact that the characteristic frequency is $\Omega_{b} \sim 1 / \ln (1 / \beta) \ll 1$. Substituting into the expression (32) we arrive at

$$
\dot{\theta}=\frac{\varepsilon}{2 \eta} \operatorname{Re}\left[\frac{E_{0 a} E_{0 b}^{*}}{1+i \Omega_{b} \ln (1 / \beta)}\right] \mathbf{u}_{y} .
$$

For electro-orientation we have

$$
\dot{\theta}=\frac{\varepsilon}{2 \eta} \frac{E_{0}^{2} \cos \theta \sin \theta}{1+\Omega_{b}^{2} \ln ^{2}(1 / \beta)}
$$




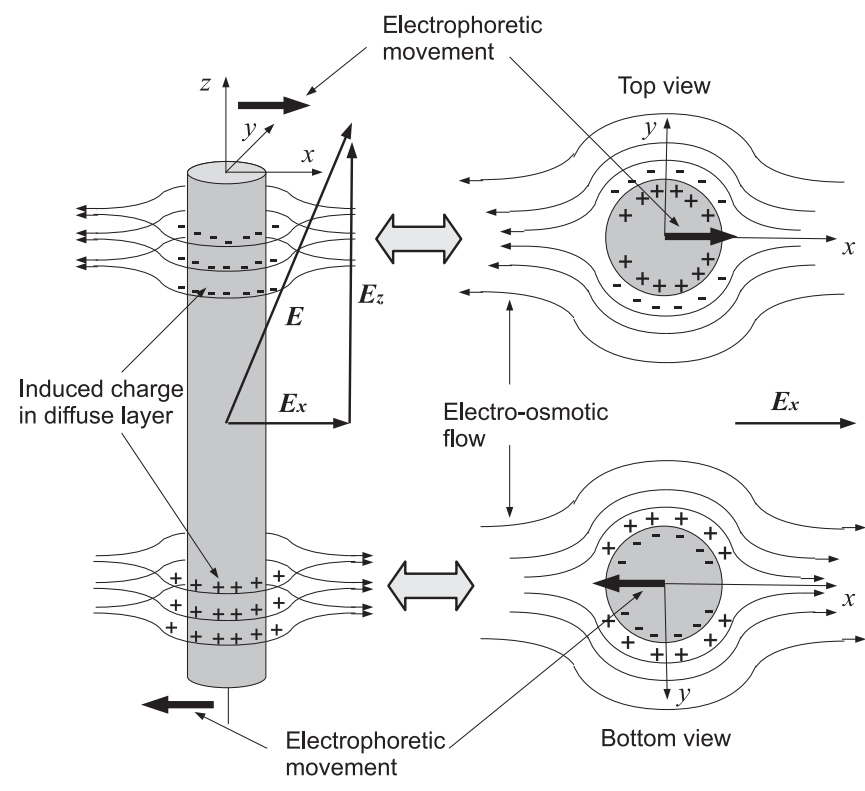

FIG. 4. Scheme of charges induced in double layer by axial electric field $E_{z}$ and electrokinetic flow generated due to the action of electric field perpendicular component $E_{x}$ on these charges.

and the particle tends to align with the field. For electrorotation we have

$$
\dot{\theta}=\frac{\varepsilon E_{0}^{2}}{2 \eta} \frac{\Omega_{b} \ln (1 / \beta)}{1+\Omega_{b}^{2} \ln ^{2}(1 / \beta)}
$$

and the particle rotates in the same direction of rotation as the electric field (co-field rotation). For both EOr and ROT, the maximum value of $\dot{\theta}$ generated by ICEO is twice the maximum value obtained from the torque on the induced dipole [expressions (24) and (26)]. In the limit $\Omega_{b} \rightarrow 0$ Eq. (36) is identical to that given in Refs. $[15,18] \dot{\theta}=$ $\varepsilon E_{0}^{2} \cos \theta \sin \theta / 2 \eta$, taking into account the time average of $\cos ^{2}(\omega t)=1 / 2$.

Figure 4 provides a qualitative explanation in terms of induced charges of the induced rotation. The arrangement of charges and flows is only for the term that produces rotation $\Phi_{s a} \nabla_{s} \Phi_{b}^{*}$. Because of symmetry, the terms $\Phi_{s a} \nabla_{s} \Phi_{a}^{*}$ and $\Phi_{s b} \nabla_{s} \Phi_{b}^{*}$ generate flow around the particle but not rotation. Figure 4 shows the induced charge in the diffuse layer due to the axial component of the electric field and the electrokinetic flow generated by the action of the electric field perpendicular component on this charge. We can imagine that the upper part of the rod is like a particle in electrophoretic motion to the right while the rod lower part is like a particle undergoing electrophoretic motion to the left. In EOr the two field components are in phase and the motion aligns the particle with the field. In ROT the field components are out of phase. The charges induced by the axial electric field take some time to develop (of the order of the RC time for charging the DL capacitance through the bulk resistor) and then the out-of-phase perpendicular field acts on these charges. The resulting particle rotation is co-field. The term $\Phi_{s a} \nabla_{s} \Phi_{a}^{*}$ is responsible for the quadrupolar flow pattern shown in Fig. 5; see Ref. [17]. This figure shows the case when the field is parallel with the major axis of the rod. The figure shows a

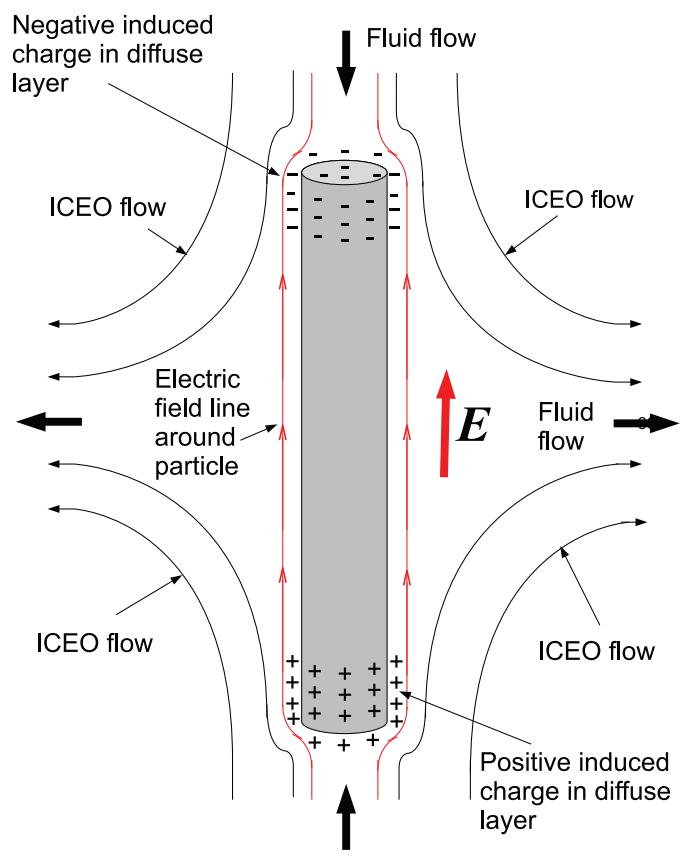

FIG. 5. (Color online) Scheme of charges induced in the diffuse layer by the axial electric field $E_{z}$ and the electrokinetic flow generated due to the action of this field on this induced charge.

scheme of the induced charge in the diffuse layer due to the axial electric field and the electrokinetic flow generated by the action of this field on this charge. This flow pattern extends at distances of the order of the length of the rod. The flow pattern generated by $\Phi_{s b} \nabla_{s} \Phi_{b}^{*}$ extends to much smaller dimensions, of the order of the rod diameter. By symmetry, the flow pattern shown in Fig. 5 does not generate any particle rotation. If the field is not parallel to the rod axis, the flow pattern is a combination of those shown in Figs. 4 and 5.

The slip velocity predicted by the standard model is higher than that measured experimentally, sometimes by an order of magnitude [30]. Several reasons for this have been proposed including dielectric coating [31-33], counter-ion crowding [34], ion adsorption [32,35], and/or surface roughness [36]. The induced voltage drop across the double layer for our nanorods is of the order of $50 \mathrm{mV}$, so that ion crowding can be disregarded. However, the other effects could play a role. To account for these effects, a phenomenological expression for the angular velocity induced on the particles can be written as $\dot{\theta}=\dot{\theta}_{\text {dipole }}+\Lambda \dot{\theta}_{\text {ICEO }}$, where $\Lambda$ is a factor (smaller than 1) which takes into account the fact that, experimentally, the slip velocity is smaller than that predicted by the standard model.

\section{EXPERIMENTS}

\section{A. Experimental details}

The silver nanowires (Nanostructured \& Amorphous Materials, Inc.) were supplied as a paste consisting of $70 \%$ wt. silver and 30\% wt. ethanol. During the synthesis of the silver nanowires, PVP (polyvinylpyrrolidone) is added and although the particles are washed several times, small amounts might remain on the silver surface. Figure 6 shows an SEM image of the nanowires, together with a scheme 


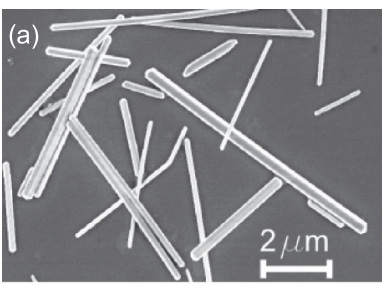

(b)

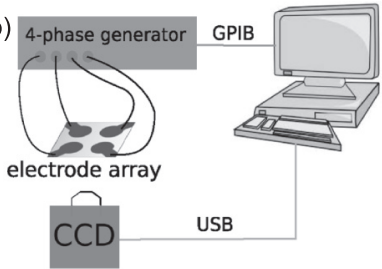

(c)
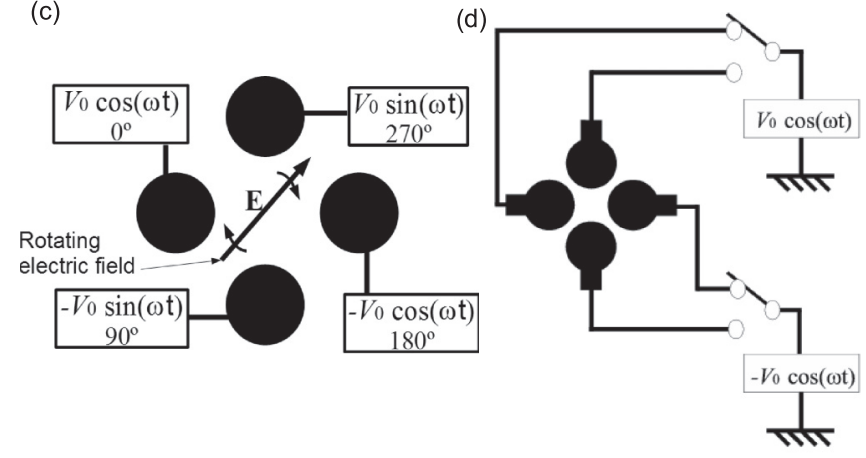

FIG. 6. (a) SEM image of silver nanowires. (b) Scheme of experimental setup. (c) Configuration for electrorotation experiments; $V_{0}$ is the applied voltage. (d) Configuration for electro-orientation experiments.

of the experimental setup and electrode. Note the different connections and applied signals for EOr and ROT experiments. Analysis of the dimensions of the silver nanowires shows that they have a range of lengths and widths. Experimental data is collected from nanowires with lengths $2 a$ ranging between 6 and $7 \mu \mathrm{m}$. The diameter $2 b$ of the wires varies between $160 \mathrm{~nm}$ and $320 \mathrm{~nm}$. A statistical study of the aspect ratio $(\beta=b / a)$ using 21 SEM images gives the aspect ratio $\beta=0.04 \pm 0.01$, where the uncertainty is given by the standard deviation in the distribution.

The nanowires were washed several times in an aqueous solution of $\mathrm{KCl}$ with a specific concentration and then suspended in $\mathrm{KCl}$ electrolyte with the same concentration. Experiments were performed using three different values of electrolyte conductivity: $1.5,5$, and $15 \mathrm{mS} / \mathrm{m}$. A sample of the nanowires was placed in the center of an electrode array made from four circularly shaped planar microelectrodes [see Figs. 6(c) and 6(d)] and the appropriate ac signal for EOr or ROT applied. The microelectrodes were made using photolithography and consisted of a $300 \mathrm{~nm}$ thick gold layer on a glass substrate. The diagonal gap between opposite electrodes was $0.5 \mathrm{~mm}$. The motion of the nanowires was observed using an inverted microscope. For ROT experiments the four electrodes were energized with a four-phase ac signal. See Fig. 6(c) for the configuration that generates a rotating field. During the application of the rotating field, the nanowires rotated counterfield; their motion was recorded with video. In EOr experiments, an ac electric field along a fixed axis was applied and the nanowires were observed to orientate along this direction. When steady state was reached, the direction of the applied field was changed by $90^{\circ}$ instantaneously. The transient orientation of the nanowires was video recorded and the procedure of changing the electric field by $90^{\circ}$ was repeated several times. The configuration for EOr is shown in Fig. 6(d). See Supplemental Material for videos of ROT and EOr [37].

The nanowire angle was determined as a function of time using a custom-made program in MATLAB. In ROT experiments, particles rotate at constant angular speed $\theta \propto t$. In EOr experiments, and according to theory [Eqs. (11) and (36)], the angular velocity is given by $\dot{\theta}=\Gamma \sin (2 \theta)$, with $\theta$ the angle between the particle axis and the applied electric field and $\Gamma$ is a constant. The integration of this equation gives

$$
\tan \theta=\tan \theta_{0} e^{-2 \Gamma t} .
$$

The experimental value of $\Gamma$ is obtained by fitting $\tan \theta(t)$ to an exponential decay.

\section{B. Experimental results}

In ROT experiments, and for any given voltage and frequency, the particles rotate counterfield at constant angular speed. In our previous publication [11], the angular speed of the wires was measured for different particle lengths and electrolyte conductivity, as a function of the applied voltage, up to $10 \mathrm{~V}_{\mathrm{pp}}$. Within this voltage range, the rotational velocity varied with the square of the voltage, as predicted by the linear model of the interaction of an electric field with the charge induced by the electric field.

Figure 7 shows ROT and EOr spectra for silver nanowires with length in the range 6 to $7 \mu \mathrm{m}$, and for three electrolyte conductivities. This size range was chosen so that several nanowires could be measured at the same time in the field of
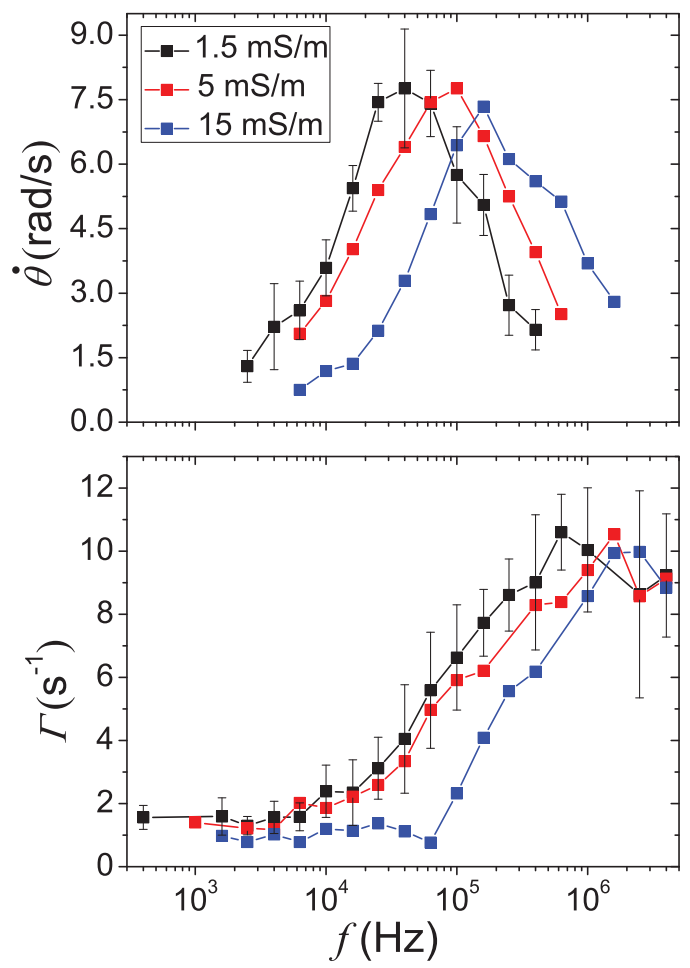

FIG. 7. (Color online) $\dot{\theta}$ (top) and $\Gamma$ (bottom) as functions of applied signal frequency for silver nanowires of length in the range 6 to $7 \mu \mathrm{m}$ and for three different electrolyte conductivities. Voltage amplitude is $10 \mathrm{~V}_{\mathrm{pp}}$. For clarity, error bars are only shown for electrolyte conductivity of $1.5 \mathrm{mS} / \mathrm{m}$. 
(a)

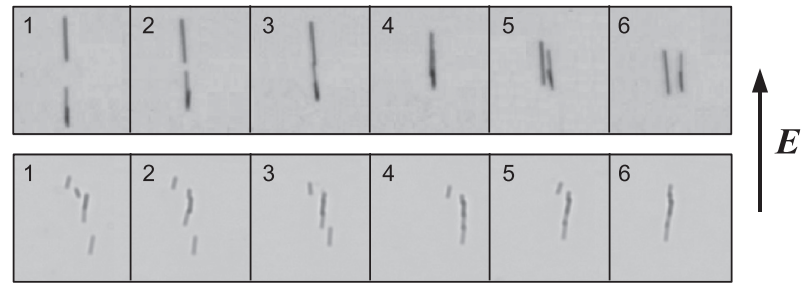

FIG. 8. Consecutive series of images showing the observed particle-particle interaction. (a) At low frequency $(3.2 \mathrm{kHz})$ the particles attract each other end to end, go side by side, and then are pushed apart from their centers. This is in accordance with the ICEO flow depicted in Fig. 5. (b) At high frequency (1.6 MHz) the particles are attracted end to end and form "pearl chains." The electrolyte conductivity was $1.5 \mathrm{mS} / \mathrm{m}$ and the applied voltage $8 \mathrm{~V}_{\mathrm{pp}}$.

view $(300 \times 225 \mu \mathrm{m})$. Each data point represents the average of at least five different nanowires and the error bars indicate the range the dispersion in these measurements. These data are collected with a constant voltage amplitude $10 \mathrm{~V}_{\mathrm{pp}}$. The angular velocity $\dot{\theta}$ as a function of signal frequency $f$ forms a bell shape with a single peak and the peak frequency of nanowire rotation increases with electrolyte conductivity.

In EOr experiments, the particle long axis aligns with the direction of the electric field. The typical time to reach alignment is given by the reciprocal of $\Gamma$; see Eq. (38). $\Gamma$ changes with the applied signal frequency $f$ and shows two plateaus (within experimental error), one at low frequencies and another at high frequencies. The plateau at low frequencies corresponds to ICEO induced orientation while the one at high frequencies corresponds to orientation of the particle from the induced-dipole torque. Experimentally, it was observed that the value of $\Gamma$ at high frequencies is independent of electrolyte conductivity (within experimental error). However, the value of $\Gamma$ at low frequencies varies with electrolyte conductivity. For example the value at the the highest conductivity is consistently smaller than for the other two electrolyte conductivities, indicating less orientation at this conductivity over this lower frequency range.

EOr experiments showed interesting particle-particle interactions whenever two nanowires were close. Figure 8(a) shows two nanowires interacting at low frequencies. The nanowires first attract each other end to end, but then move along side by side and are finally repelled from each other. Figure 8(b) shows several nanowires at high frequencies. The wires attract each other end to end and form "pearl chains." This behavior of metal particles at low frequencies was first observed by Rose et al. [17] as ICEO flow causing particle-particle interaction. It is driven by the ICEO quadrupolar flow pattern shown in Fig. 5, where liquid is driven into the two ends of the particle and pushed away from the center. The observation of pearl-chaining behavior at high frequencies is typical of dipole-dipole interactions $[12,13]$.

\section{DISCUSSION}

In a previous work we compared our ROT measurements of perfectly polarizable metal cylinders with dipole theory [11] and good agreement between theory and experiment was found. Therefore, we start by comparing our ROT and EOr
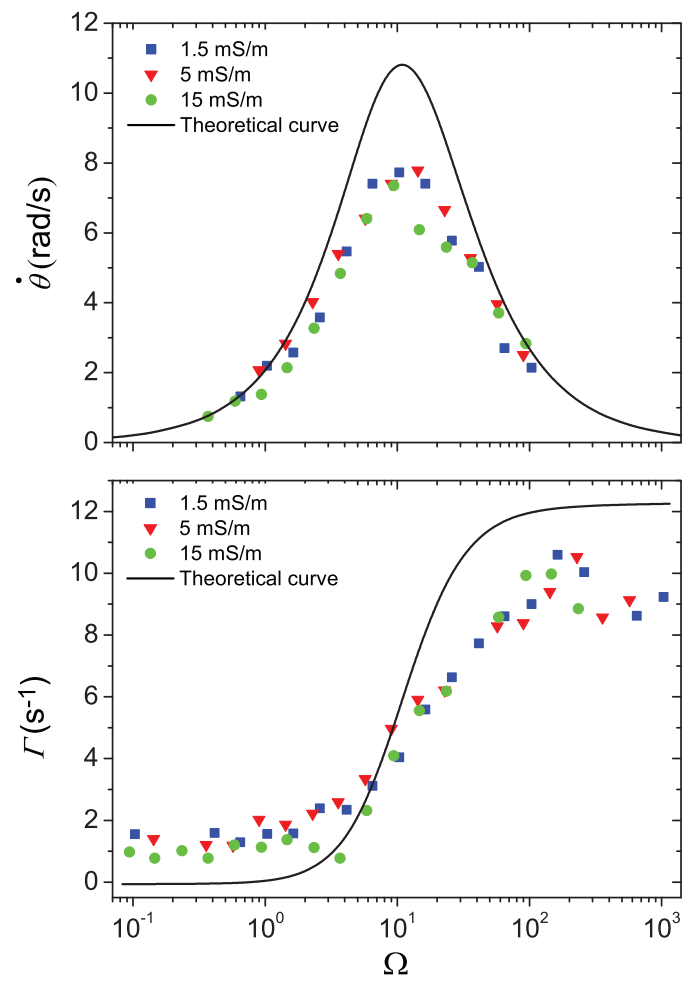

FIG. 9. (Color online) ROT (top) and EOr (bottom) spectra as function of nondimensional frequency.

measurements with dipole theory as summarized in Fig. 9. Figure 9 (top) shows experimental and theoretical ROT speed as a function of nondimensional frequency $\Omega=\omega a C_{\mathrm{DL}} / \sigma$ (i.e., ROT spectra). The theoretical curve is obtained for rods with lengths $2 a=6.5 \mu \mathrm{m}$ and aspect ratio $\beta=0.04$. Also for the theoretical curve, $E^{2}$ was computed for the experimental electrode array using COMSOL from the average of the torque within the field of view giving $E^{2}=112 \times 10^{6} \mathrm{~V}^{2} / \mathrm{m}^{2}$. In order to nondimensionalize the experimental frequency, the ROT data were fitted to a Lorentzian to obtain the best fit values for the frequency of the maximum $\omega_{\mathrm{RC}}$, and from here the specific capacitance $C_{\mathrm{DL}}$ for each electrolyte conductivity was derived.

Table I shows these capacitance values. Also shown are the theoretical values for the diffuse layer capacitance calculated from the Debye-Hückel theory, determined from $C_{\mathrm{DH}}=\varepsilon / \lambda_{D}$ where $\lambda_{D}$ is the Debye length of the electrolyte. Experimental capacitance values are quite close to the theoretical DebyeHückel values, supporting our hypothesis that the particle

TABLE I. Values for the electrical double layer capacitance obtained from experiments $\left(C_{\mathrm{DL}}\right)$, Debye-Hückel theoretical values for the Debye layer $\left(C_{\mathrm{DH}}\right)$, and best-fit values for Debye and compact layers in series $\left(C_{\mathrm{S}+\mathrm{DH}}\right)$.

\begin{tabular}{lccc}
\hline \hline $\begin{array}{l}\text { Conductivity } \\
(\mathrm{mS} / \mathrm{m})\end{array}$ & $C_{\mathrm{DL}}\left(\mathrm{F} \mathrm{m}^{-2}\right)$ & $C_{\mathrm{DH}}\left(\mathrm{F} \mathrm{m}^{-2}\right)$ & $C_{\mathrm{S}+\mathrm{DH}}\left(\mathrm{F} \mathrm{m}^{-2}\right)$ \\
\hline 1.5 & $1.9 \times 10^{-2}$ & $2.3 \times 10^{-2}$ & $1.9 \times 10^{-2}$ \\
5 & $3.5 \times 10^{-2}$ & $4.2 \times 10^{-2}$ & $3.0 \times 10^{-2}$ \\
15 & $4.3 \times 10^{-2}$ & $7.3 \times 10^{-2}$ & $4.4 \times 10^{-2}$ \\
\hline \hline
\end{tabular}


polarization is due to charging of the electrical double layer. Adding a Stern or compact layer in series with the DebyeHückel capacitances reduces the error further giving an overall difference of less than $15 \%$, with $C_{\mathrm{S}}=0.11 \mathrm{~F} \mathrm{~m}^{-2}$. This calculation indicates that the PVP layer which may exist on the particle surface is probably insignificant in terms of its contribution to the surface impedance.

Figure 9 (bottom) shows experimental and theoretical EOr exponential parameter $\Gamma$ as a function of nondimensional frequency $\Omega=\omega a C_{\mathrm{DL}} / \sigma$ (i.e., EOr spectra). The specific capacitances obtained from the ROT experiments were used to nondimensionalize the frequency. To calculate the theoretical curve for EOr, the electric field was determined from the average value of the torque on particles within the field of view equal to $E^{2}=121 \times 10^{6} \mathrm{~V}^{2} / \mathrm{m}^{2}$. Inspection of the data shows that dipole theory can qualitatively explain the frequency dependence of the EOr. However, it is apparent that dipole theory cannot explain the nonzero value of the orientation at low frequencies because dipole theory predicts that the orientation should be negligible for slender cylinders. This apparent difference can be ascribed to orientation due to ICEO flow around the particle as shown schematically in Fig. 4. In support of this hypothesis, Rose et al. [17] showed that particle-particle interaction followed the behavior expected from the electrokinetic flow pattern shown in Fig. 5 and from the experimental images in Fig. 8(a). In addition, as the electrolyte ionic strength increases the ICEO flow will reduce, as seen in the data, and as reported for other experimental systems [30,38,39]. At high frequencies the ICEO flow is negligible and particle orientation is solely due to the dipole torque.

As shown in Fig. 9 the experimental values of $\Gamma$ at high frequencies are lower than predicted (the mean experimental value at high frequencies is 0.78 times the theoretical value). The discrepancy can be explained as an enhancement of the viscous friction near a wall. In ROT, the experimental values of the angular rotation speed are also lower than the theoretical values (mean experimental peak is 0.70 times the theoretical). These two factors $(0.78$ and 0.70$)$ are very similar reinforcing the hypothesis that the ROT values are mainly due to the dipole torque as outlined previously [11]. Note that EOr values are only due to dipole torque at high frequencies. In addition, the difference in the two values ( 0.78 in EOr to 0.70 in ROT) is consistent with the existence of a small amount of co-field rotation in ROT experiments due to ICEO, which would lead to a small decrease in the total angular rotation.

To estimate the viscous friction near a wall we use the expression for the viscous friction per unit length of an infinite cylinder moving parallel to a wall [40],

$$
F=-\frac{4 \pi \eta}{\cosh ^{-1}(h / b)} U
$$

where $F$ is the force per unit length, $U$ is the velocity of the cylinder, and $h$ is the distance from the cylinder axis to the wall. For a slender finite cylinder and neglecting end effects, the approximate expression of the rotational friction coefficient is obtained from $U=\dot{\theta} z$, where $z$ is the coordinate along the cylinder axis measured from its center. The rotational viscous coefficient is then [41]

$\gamma=\frac{1}{\dot{\theta}} \int_{-a}^{a} z(-F) d z=\frac{8 \pi \eta a^{3}}{3 \cosh ^{-1}(h / b)}=\gamma_{\infty} \frac{f(\lambda)}{\cosh ^{-1}(h / b)}$,

where $\gamma_{\infty}$ is the coefficient in the bulk and $f(\lambda)$ is the Broersma function defined previously [Eq. (9)]. The typical height $h$ of the nanorods above the electrode surface can be determined by considering that they are Brownian particles. The typical fluctuation of gravitational energy $\Delta \rho \tau g \Delta h$ is equal to the thermal energy $k_{B} T$ at room temperature, so that $\Delta h=\left(k_{B} T\right) /(\Delta \rho \tau g \Delta h)$, where $\Delta \rho$ is the difference in densities between silver and water, $\tau$ is the volume of a nanorod, and $\Delta h$ is the increment of $h$. This gives an estimate of $\Delta h=0.12 \mu \mathrm{m}$ for the nanorods. Inserting this value into the expression gives $\gamma \sim 1.9 \gamma_{\infty}$. According to this estimate angular speed values near the wall would be around 0.5 times smaller than the values in the bulk. The observed reduction in the EOr of 0.78 would be obtained if $\Delta h \approx 0.3 \mu \mathrm{m}$, which is consistent with the above calculations (on the same order as the estimate of $\Delta h=0.12 \mu \mathrm{m})$.

A further correction comes from the change in the electrical torque near a wall. For a sphere rotating in contact with an insulating wall we have previously calculated a reduction of the electrical torque of around $6.5 \%[9,10]$.

The slender theory for EOr and ROT outlined in this paper shows that the expected particle rotation due to ICEO flow and particle rotation due to induced-dipole torque (IDT) have the same characteristic frequency. While ICEO driven rotation decreases with frequency, IDT rotation increases, so that the two spectra follow opposite trends. Taking the limiting experimental values for low and high frequencies, it is possible to construct a phenomenological curve for EOr as a function of frequency from

$$
\Gamma=\Gamma_{0} \frac{\Omega_{m}^{2}}{\Omega_{m}^{2}+\Omega^{2}}+\Gamma_{\infty} \frac{\Omega^{2}}{\Omega_{m}^{2}+\Omega^{2}},
$$

where $\Gamma_{0}$ and $\Gamma_{\infty}$ are the $\Gamma$ values for zero and infinite frequency, respectively. Figure 10 shows and example of this phenomenological curve for one electrolyte (conductivity

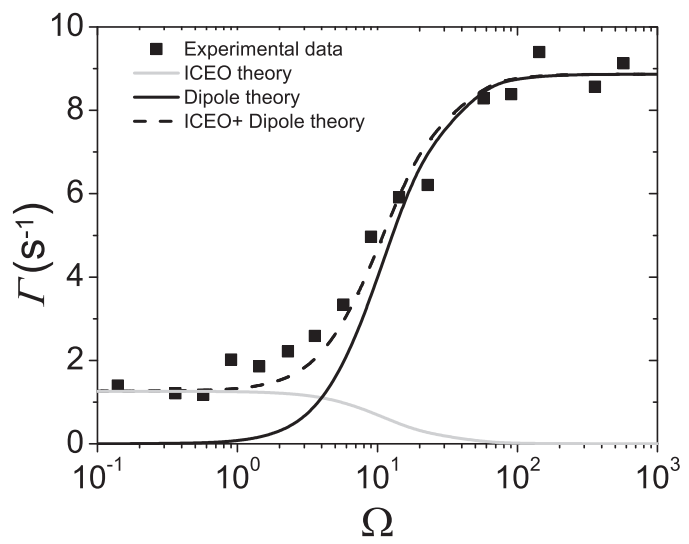

FIG. 10. ICEO and dipole contributions to the observed $\Gamma$ as a function of frequency in EOr experiments. The curve is for electrolyte with conductivity $5 \mathrm{mS} / \mathrm{m}$. 
$\sigma=5 \mathrm{mS} / \mathrm{m}$ ) together with the corresponding experimental values. The figure shows that the experimental values can be fitted to a curve given by Eq. (41) with a characteristic frequency obtained from the ROT experiments.

Electro-orientation of metal nanowires shows some similarities with the motion of polarizable metal particles in diverging ac fields. In general, the real part of the induced dipole governs the frequency dependence of the dielectrophoretic (DEP) force on a particle. In addition, particle motion can be driven by electro-osmotic flow around the particle. Induced-charge electrophoresis (ICEP) refers to the particle motion driven by ICEO flow around the particle [21]. A theory describing the frequency-dependent motion of a polarizable metal sphere in a diverging ac field as a combination of ICEP and DEP was presented by Miloh [42]. The ICEP is frequency dependent with approximately the same relaxation frequency as the real part of the dipole, disappearing at frequencies much higher than the relaxation of the induced dipole [42]. ICEP for a metal sphere has the same qualitative dependence on frequency as the ICEO EOr for metal rods as shown schematically in Fig. 10. For spheres with thin double layers and at low frequencies the prediction [42] is that the ICEP and DEP are of similar magnitude but in opposite directions. Theory shows that DEP is negative at low frequencies and positive at high frequencies, while ICEP is maximum at zero frequency and decreases with frequency. The characteristic frequency is of the order of the reciprocal of the RC time for charging the double-layer capacitance of the sphere. For zero frequency, ICEP and DEP cancel and there is no particle movement, as discussed also in Refs. [14,43]. However, experimentally this behavior has not been observed. In a previous publication, we observed negative DEP at low frequencies for metal spheres with rough surfaces [9]. These results imply that ICEP is lower than predicted by the model that assumes an ideal double layer. As demonstrated in this paper similar behavior is seen for orientation of rods, where the ICEO-EOr is small but not zero so that orientation is observed at low frequencies. Figures 9 and 10 show the existence of ICEO flow (for metal rods) but this flow is also lower than predicted by the standard model. The predicted theoretical ratio between ICEO and IDT angular velocities can be obtained from Eqs. (24) and (36), and is a factor of 2. Therefore, from the measured values of $\Gamma$ at low frequencies as seen in Figs. 9 and 10, the ICEO flow is at least a factor of 10 smaller than predicted. On the other hand, the magnitude and frequency of the electrical torque on the induced dipole is close to that predicted by dipole theory presented in this paper.

\section{CONCLUSIONS}

A comprehensive theoretical and experimental study of the ac electrokinetic properties of metallic rods in the thin double layer approximation have been presented. The angular rotation of nanorods induced by ac electric fields is governed by both induced-dipole torque (IDT) on the particle and induced-charge electro-osmotic (ICEO) flow around the particle.

In ROT experiments, both mechanisms occur in the same range of frequencies, of the order of the characteristic frequency $\omega_{R C}$ for charging the DL capacitor through a resistive medium, the electrolyte. The IDT generates a counterfield rotation while the ICEO flow generates a co-field rotation. Both mechanisms result from the interaction of the out-of-phase induced charge with the applied electric field, which means that they both mirror the imaginary part of the particle polarizability. Experiments show counterfield rotation close to the dipole theory prediction indicating that the ICEO flow is much smaller than expected from standard ICEO theory. This is common in ICEO experiments and several effects have been proposed that tend to diminish the ICEO flow, such as dielectric coating, surface roughness, ion adsorption, and/or ion crowding.

In EOr experiments, the two mechanisms occur in different ranges of frequencies with an overlap region around $\omega_{\mathrm{RC}}$. Both mechanisms tend to align the rod with the direction of the applied electric field. ICEO EOr is dominant for frequencies $\omega \ll \omega_{\mathrm{RC}}$, while IDT EOr is dominant for $\omega \gg \omega_{\mathrm{RC}}$. Both mechanisms result from the interaction of the in-phase induced charge with the applied electric field, which means that they both mirror the real part of the particle polarizability. The characteristic angular speed $\Gamma$ is much greater for IDT EOr than for ICEO EOr indicating again that ICEO flow is much smaller than expected from standard theory. From the measured value of $\Gamma$ at low frequencies, the ICEO flow is at least a factor of 10 smaller than predicted. On the other hand, the magnitude and frequency of the electrical torque on the induced dipole is close to that predicted by dipole theory presented in this paper.

EOr of metal rods presents some similarities with DEP of metal particles. Both phenomena result from the interaction of the in-phase induced charge with the applied electric field. This interaction results in two distinct mechanisms for generating particle motion: induced-dipole interaction with field (maximum at high frequency) and induced-charge electro-osmotic flow around the particle (maximum at low frequency).

The present work provides a zero-order framework to predict the behavior of metallic particles suspended in electrolytes in ac fields. Given the increasing level of interest in the use of ac electrokinetic techniques to manipulate and assemble

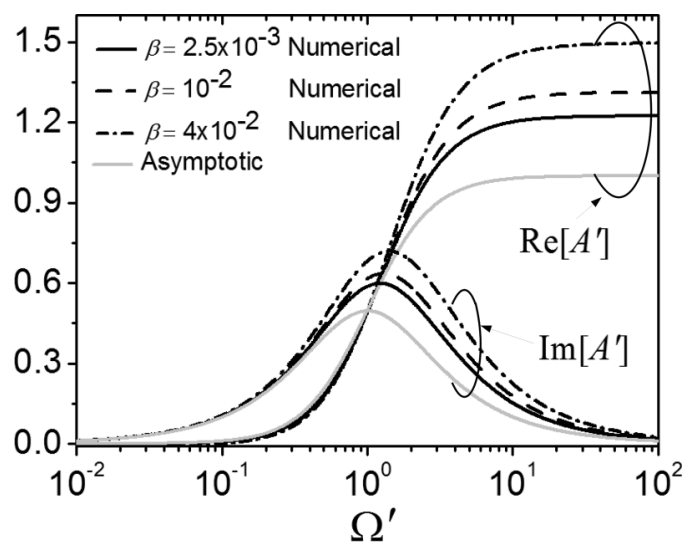

FIG. 11. Nondimensional polarizability $A^{\prime}=3 A \ln (1 / \beta)$ as a function of nondimensional frequency $\Omega^{\prime}=\Omega \beta \ln (1 / \beta)$ from the numerical solutions and from the analytical slender-body approximation. The numerical solutions approach the analytical solution as $\beta \rightarrow 0$. 
nanoparticles and nanowires a full theoretical understanding will enable further development of this technology for sensors and nanoelectronics. There are many publications that explore the ac electrokinetics of metal nanoparticles where the behavior of the particles is modeled using Maxwell-Wagner polarization, ignoring the double layer. This leads to erroneous predictions and, in particular, cannot explain the experimental results presented here and in our previous publications.

\section{ACKNOWLEDGMENTS}

We acknowledge financial support from Junta de Andalucía (Contract No. P09-FQM-4584) and the Spanish Government Ministry MEC (Contract No. FIS2011-25161).

\section{APPENDIX}

Figure 11 shows a comparison of the results of the complex polarizability $\alpha_{z}$ of cylinders obtained using numerical com- putations and the slender-body analytical solution. The figure shows the real and imaginary parts of a nondimensional polarizability $A^{\prime}=3 \alpha_{z} \ln (1 / \beta) / 4 \pi \varepsilon a^{3}=3 A \ln (1 / \beta)$ as a function of a nondimensional frequency $\Omega^{\prime}=\omega C_{\mathrm{DL}} b \ln (1 / \beta) / \sigma=$ $\Omega \beta \ln (1 / \beta)$. With these definitions, the slender-body analytical solution for $A^{\prime}$ as a function of $\Omega^{\prime}$ is universal (it does not depend on aspect ratio $\beta$ ). The figure shows that the numerical solutions approach the analytical solution as $\beta$ goes to zero, although slowly as corresponds to a small parameter that decreases as $1 / \ln (1 / \beta)$. The analytical results provide the following scaling for the polarizability and frequency, respectively:

$$
\alpha_{z}=\frac{4 \pi a^{3} \varepsilon}{3 \ln (a / b)} A^{\prime}, \quad \omega=\frac{\sigma}{C_{\mathrm{DL}} b \ln (a / b)} \Omega^{\prime},
$$

where $A^{\prime}$ and $\Omega^{\prime}$ are of order unity as $\beta=b / a$ changes.
[1] Y. Cui, Q. Wei, H. Park, and C. M. Lieber, Science 293, 1289 (2001).

[2] Y. Li, F. Qian, J. Xiang, and C. M. Lieber, Mater. Today 9(10), 18 (2006).

[3] M. Law, L. E. Greene, J. C. Johnson, R. Saykally, and P. Yang, Nat. Mater. 4, 455 (2005).

[4] J. J. Boote and S. D. Evans, Nanotechnology 16, 1500 (2005).

[5] B. C. Gierhart, D. G. Howitt, S. J. Chen, R. L. Smith, and S. D. Collins, Langmuir 23, 12450 (2007).

[6] L. Zhang and Y. Zhu, Appl. Phys. Lett. 96, 141902 (2010).

[7] D. L. Fan, F. Q. Zhu, X. Xu, R. C. Cammarata, and C. L. Chien, Proc. Natl. Acad. Sci. USA 109, 9309 (2012).

[8] Y. Ren, D. Morganti, H. Y. Jiang, A. Ramos, and H. Morgan, Langmuir 27, 2128 (2011).

[9] P. García-Sánchez, Y. Ren, J. J. Arcenegui, H. Morgan, and A. Ramos, Langmuir 28, 13861 (2012).

[10] J. J. Arcenegui, A. Ramos, P. García-Sánchez, and H. Morgan, Electrophoresis 34, 979 (2013).

[11] J. J. Arcenegui, P. García-Sánchez, H. Morgan, and A. Ramos, Phys. Rev. E 88, 033025 (2013).

[12] T. B. Jones, Electromechanics of Particles (Cambridge University Press, Cambridge, UK, 2005).

[13] H. Morgan and N. G. Green, AC Electrokinetics: Colloids and Nanoparticles (Research Studies Press Ltd., Hertfordshire, England, 2003).

[14] T. M. Squires and M. Z. Bazant, J. Fluid Mech. 560, 65 (2006).

[15] D. Saintillan, E. Darve, and E. S. G. Shaqfeh, J. Fluid Mech. 563, 223 (2006).

[16] K. A. Rose, J. A. Meier, G. M. Dougherty, and J. G. Santiago, Phys. Rev. E 75, 011503 (2007).

[17] K. A. Rose, B. Hoffman, D. Saintillan, E. S. G. Shaqfeh, and J. G. Santiago, Phys. Rev. E 79, 011402 (2009).

[18] E. Yariv, J. Fluid Mech. 613, 85 (2008).

[19] A. Ramos, H. Morgan, N. G. Green, and A. Castellanos, J. Colloid Interface Sci. 217, 420 (1999).

[20] A. Ajdari, Phys. Rev. E 61, R45 (2000).

[21] M. Z. Bazant and T. M. Squires, Phys. Rev. Lett. 92, 066101 (2004).
[22] N. G. Green and T. B. Jones, J. Phys. D 40, 78 (2007).

[23] S. Broersma, J. Chem. Phys. 32, 1626 (1960).

[24] S. Broersma, J. Chem. Phys. 74, 6989 (1981).

[25] H. A. Stone, J. R. Lister, and M. P. Brenner, Proc. R. Soc. London A 455, 329 (1999).

[26] M. M. Hohman, M. Shin, G. Rutledge, and M. P. Brenner, Phys. Fluids 13, 2201 (2001).

[27] E. J. Hinch, Perturbation Methods (Cambridge University Press, Cambridge, UK, 1991).

[28] A. González, A. Ramos, N. G. Green, A. Castellanos, and H. Morgan, Phys. Rev. E 61, 4019 (2000).

[29] Y. Solomentsev and J. L. Anderson, J. Fluid Mech. 279, 197 (1994).

[30] M. Z. Bazant and T. M. Squires, Curr. Opin. Colloid Interface Sci. 15, 203 (2010)

[31] N. G. Green, A. Ramos, A. González, H. Morgan, and A. Castellanos, Phys. Rev. E 66, 026305 (2002).

[32] A. J. Pascall and T. M. Squires, Phys. Rev. Lett. 104, 088301 (2010).

[33] M. S. Kilic and M. Z. Bazant, Electrophoresis 32, 614 (2011).

[34] M. Z. Bazant, M. S. Kilic, B. D. Storey, and A. Ajdari, Adv. Colloid Interface Sci. 152, 48 (2009).

[35] Y. K. Suh and S. Kang, Phys. Rev. E 79, 046309 (2009).

[36] R. J. Messinger and T. M. Squires, Phys. Rev. Lett. 105, 144503 (2010).

[37] See Supplemental Material at http://link.aps.org/supplemental/ 10.1103/PhysRevE.88.063018 for videos of electrorotation and electro-orientation of silver nanowires.

[38] N. G. Green, A. Ramos, A. González, H. Morgan, and A. Castellanos, Phys. Rev. E 61, 4011 (2000).

[39] V. Studer, A. Pépin, Y. Chen, and A. Ajdari, Analyst 129, 944 (2004).

[40] D. J. Jeffrey and Y. Onishi, Q. J. Mech. Appl. Math. 34, 129 (1981).

[41] A. J. Hunt, F. Gittes, and J. Howard, Biophys. J. 67, 766 (1994).

[42] T. Miloh, Phys. Fluids 21, 072002 (2009).

[43] V. N. Shilov and T. S. Simonova, Colloid J. USSR 43, 90 (1981). 